\title{
Millimeter-wave survey of molecular clouds around the W5-East triggered star-forming region
}

\author{
T. Niwa ${ }^{1, \star}$, K. Tachihara ${ }^{2}$, Y. Itoh ${ }^{1}$, Y. Oasa ${ }^{1}$, K. Sunada ${ }^{3}$, K. Sugitani ${ }^{4}$, and T. Mukai ${ }^{1}$ \\ 1 Graduate School of Science and Technology, Kobe University, 1-1, Rokkodai, Nada, Kobe, Hyogo 657-8501, Japan \\ e-mail: niwa@nhao.go.jp \\ 2 National Astronomical Observatory of Japan, 2-21-1, Osawa, Mitaka, Tokyo 181-8588, Japan \\ 3 Mizusawa VERA Observatory, National Astronomical Observatory of Japan, 2-12, Hoshi-ga-oka, Mizusawa-ku, Oshu-shi, \\ Iwate 023-0861, Japan \\ 4 Graduate School of Natural Sciences, Nagoya City University, Mizuho, Mizuho, Nagoya, Aichi 467-8501, Japan
}

Received 1 October 2008 / Accepted 16 March 2009

\begin{abstract}
Aims. We examined the physical properties of molecular clouds (morphology, column density, number density, mass) to investigate the mechanism of triggered star formation by UV radiation from a massive star.

Methods. We made extensive, high-resolution maps of molecular clouds associated and interacting with the W5-East $\mathrm{H}$ II region using the $45-\mathrm{m}$ telescope at the Nobeyama Radio Observatory $\left(H P B W=15^{\prime \prime} \cdot 6\right)$ in ${ }^{13} \mathrm{CO}(J=1-0)$ and $\mathrm{C}^{18} \mathrm{O}(J=1-0)$ to reveal details in the high-density regions of the molecular clouds. In addition, to investigate the spatial distributions of young stellar objects (YSOs) in the W5-East H II region, we mapped the spatial distributions of Class I and II candidates.

Results. We identified eight ${ }^{13} \mathrm{CO}$ molecular clouds (three of them are known bright-rimmed clouds) and nine $\mathrm{C}^{18} \mathrm{O}$ clumps. The masses of the clouds and clumps range from 460 to $36000 M_{\odot}$ and from 55 to $740 M_{\odot}$, respectively. The peak ${ }^{13} \mathrm{CO}$ column densities of the clouds facing the $\mathrm{H}$ II region are twice as large as the others. They have steep density gradients toward the $\mathrm{H}$ II region, indicating interactions with the $\mathrm{H}$ II region. We selected 55 Class I candidates and 778 Class II candidates associated with the W5-East H II region from the previous Spitzer IRAC/MIPS survey. Most Class I candidates are located around integrated intensity peaks of ${ }^{13} \mathrm{CO}$, whereas most Class II candidates are distributed along the front sides of the BRC arcs close to the exciting star.

Conclusions. The alignments of the YSO candidates and the molecular clouds in order of age indicate that triggered star formation occurs in the W5-East $\mathrm{H}$ II region as a result of gas compression by strong UV radiation. Based on the column densities of ${ }^{13} \mathrm{CO}$ and the spatial distribution of YSO candidates, we identified a new bright-rimmed cloud candidate on the west side of the W5-East H II region.
\end{abstract}

Key words. ISM: clouds - ISM: H II regions - stars: evolution - stars: formation - radio lines: ISM

\section{Introduction}

\subsection{Triggered star formation}

Star formation, which occurs as a result of the condensation and gravitational collapse of molecular clouds (e.g., Shu 1977), has two major modes: spontaneous and triggered star formation. Spontaneous star formation occurs in molecular clouds in a quiescent environment, i.e., an environment with no external disturbances. In triggered star formation, molecular clouds are compressed from the outside by, for example, shock waves of supernova explosions, stellar winds, and ultraviolet radiation from OB stars.

The Taurus molecular cloud is a representative example of a spontaneous star-forming region. Numerous observational results demonstrate that this cloud is the site of low-mass star formation. The Orion molecular cloud is a representative triggered star-forming region. OB associations are distributed across 150 square degrees. The age distribution shows that young stars tend to be located close to the Orion molecular cloud (e.g., Warren \& Hesser 1978). Okumura et al. (2000) observed the W51 H II region in the near-infrared to search for young stellar

\footnotetext{
* Present address: Nishi-Harima Astronomical Observatory, Sayocho, Hyogo, 679-5313, Japan.
}

objects (YSOs) and confirmed the age alignment of the YSOs with an age distribution of 1-2 Myr.

Previous studies confirmed that molecular clouds around the $\mathrm{H}$ II region form stars with a wide mass range. For example, Yamaguchi et al. (1999) surveyed molecular clouds around $23 \mathrm{H}$ II regions in the southern hemisphere using ${ }^{13} \mathrm{CO}$ molecular line emission. They compared the luminosity-mass ratio of 204 IRAS point sources with their associated molecular masses. They discovered that the ratios for objects located on the side of the clouds near the interacting $\mathrm{H}$ II regions were an order of magnitude larger than in molecular clouds located at the far side of the H II region. This strongly indicates that molecular clouds facing an $\mathrm{H}$ II region tend to be massive star-forming regions. Deharveng et al. (2005) selected high-mass star-forming regions around Galactic H II region candidates in the Galactic $\mathrm{H}$ II regions. They pointed out that the ring-like structures in the Midcource Space eXperiment (MSX) A-band $(8.28 \mu \mathrm{m})$ suggest compressed layers of molecular clouds. Some H II regions that have ring-like structures in the MSX A-band (e.g., Deharveng et al. 2006) were found to be high-mass star-forming regions. Turning to low-mass star formation, some previous studies confirmed sequential alignments of low-mass YSOs and molecular clouds in order of age (e.g., Sugitani et al. 1995). These observations indicate evidence of triggered star formation of low-mass 
stars, summarized as Small-Scale Sequential Star Formation (reviewed by Ogura 2006). In addition, as a theoretical approach, Whitworth \& Zinnecker (2004) suggested that the UV destruction of molecular clouds is a possible formation mechanism of brown dwarfs and free-floating planetary-mass objects, many of which were discovered in H II regions (e.g., Sh-2 106, Oasa et al. 2006).

In many cases, triggered star-forming regions are associated with clusters (e.g., Elmegreen \& Lada 1977). As most field stars are believed to form as stellar cluster members (e.g., Lada \& Lada 2003), the triggered star formation process is an important mechanism for understanding the evolution of stars in the Galaxy.

\subsection{Bright rimmed clouds}

Bright-rimmed clouds (BRCs) are considered triggered star forming regions (Sugitani et al. 1991; Sugitani \& Ogura 1994). According to Sugitani et al. (1991), BRCs' selection criteria are as follows: (1) the H II region is $60^{\prime}$ or more in apparent extent and the $\mathrm{H}$ II region contacts molecular clouds; (2) the boundaries of the HII region and the molecular cloud are seen as arc-like structures in the Palomar Observatory Sky Survey (POSS) or on ESO plates; (3) protostellar IRAS point sources are located in the molecular clouds and are detected in $25 \mu \mathrm{m}$ and at least one other band. Based on these criteria, they cataloged 44 and 45 IRASassociated BRCs in the northern and southern hemispheres, respectively. This is known as the "BRC catalog" or "SFO catalog". Because the BRCs are molecular clouds whose surfaces are exposed to H II regions, they are expected to show triggered star formation of various mass objects (e.g., Deharveng \& Zavagno 2008). Urquhart et al. (2007) carried out a multi-wavelength study of SFO 75 showing two fragmented cores perpendicular to the surface of the expanding HII region. Moreover, in SFO 75, a reddened star was detected only on the near-side core of the $\mathrm{H}$ II region by Spitzer GLIMPSE observations. This is one type of observational evidence of high-mass star formation in BRC. In a resent study, Morgan et al. (2008) surveyed 44 BRCs in the sub-millimeter wavelength $(450 \mu \mathrm{m}, 850 \mu \mathrm{m})$ using SCUBA mounted on the James Clerk Maxwell Telescope (JCMT). They conclude that 42 of 44 BRCs have dense dust cores whose density is about $10^{5} \mathrm{~cm}^{-3}$ on average. The mass range of these cores is from 0.03 to $50 M_{\odot}$. They also discussed the relationships between core mass function and BRC morphologies. According to their studies younger-phase BRCs tend to have steeper core mass functions.

\subsection{W5-East}

The W5 HII region is part of the W3/W4/W5 cloud complex (Carpenter et al. 2000) located at $2 \mathrm{kpc}$ (Georgelin \& Georgelin 1976). The W5-East H II region (hereafter W5-East) is the eastern part of the W5 H II region, and is excited mainly by $\mathrm{BD}+590578(\mathrm{O} 7 \mathrm{~V})$. The molecular clouds around the HII region are assumed to be at the same distance as BD +590578 . This is a well studied region to investigate triggered star formation caused by UV radiation because it has such a simple structure: all molecular clouds in the H II region are the same distance from the exciting star. This indicates that the molecular clouds have the same ionizing conditions (i.e., radiation field, excitation temperature, etc.).

Previous studies include observations of the W5-East region in the millimeter-wave band. Wilking et al. (1984) carried out a molecular cloud survey toward the dark clouds W5NE and W5NW. They observed ${ }^{12} \mathrm{CO}(J=1-0)$ and ${ }^{13} \mathrm{CO}(J=$ 2-1) with the Millimeter Wave Observatory 4.9-m telescope $(F W H M=2 ! 3)$. They revealed molecular cloud distributions and showed that W5NE is excited by a zero-age main-sequence star (ZAMS) inside the molecular cloud. W5 was also observed in ${ }^{12} \mathrm{CO}(J=1-0)$ by the FCRAO $14-\mathrm{m}$ telescope with a spatial resolution of $50^{\prime \prime}$, as part of the Canadian Galactic Plane Survey (CGPS, Heyer et al. 1998). This unbiased survey revealed the distribution of low-density gas in W5-East. Carpenter et al. (2000) carried out observations in the near-infrared $K$-band and ${ }^{13} \mathrm{CO}(J=1-0)$ around 32 IRAS point sources in the W3/W4/W5 cloud complex. About 1600 stars were identified from the $K$-band observations, half of which are associated with five IRAS point sources and form rich clusters. In addition, $39 \%$ of the cluster members are associated with the molecular clouds, with about $130 M_{\odot}$ on average. One of these is known as the AFGL 4029 IR cluster. Karr \& Martin (2003) discussed triggered star formation in W5 using multi-wavelength archival data. From the expansion of W5-East and the age of observed YSOs, they concluded that the timescale of interaction between the molecular clouds and the $\mathrm{H}$ II region was 0.5-1 Myr.

Matsuyanagi et al. (2006) carried out a deep near-infrared survey of BRC 14 that identified 74 YSO candidates with a lower limit on mass detection of $0.12 M_{\odot}$. They investigated starformation activities using three indicators (object extinction, ratio of YSOs to detected objects, and YSO infrared excess) and found that all parameters increased away from the exciting star. They concluded that triggered star formation of low-mass stars occurred in BRC 14. This is the first observational evidence for triggered star formation down to sub-solar mass stars. Recent observations with the Spitzer Space Telescope showed embedded clusters of Class I sources in the molecular cloud, whereas Class II sources are more widely distributed (Koenig et al. 2008). They pointed out triggered star formation in BRC 13 and 14 from the Class I/II sources distributions.

In spite of these observational indications of triggered star formation, no high-resolution extensive cloud and dense-clump surveys exist to compare directly with the infrared YSOs. The ${ }^{13} \mathrm{CO}$ molecular cloud survey (e.g., Carpenter et al. 2000) area is limited around the IRAS point source $\left(6{ }^{\prime} .2 \times 4.8\right)$, thus we cannot reveal relationships between the star formation activity and the large-scale triggering effect by W5-East. Moreover, no observation using denser gas tracers (e.g., $\mathrm{C}^{18} \mathrm{O}$ ) have been carried out in this region. To investigate detailed cloud structures of interest interacting between clouds and $\mathrm{H}$ II regions and triggered star formation, we performed an unbiased, high spatial resolution survey of the entire W5-East region. Derived physical properties of clouds and clumps such as morphology, density, mass, and association with bright rims were compared in terms of star-formation activities based on the Spitzer IRAC/MIPS survey $(3.5,5.8,8.0$, and $24.0 \mu \mathrm{m})$ by Koenig et al. (2008).

\section{Observations and analysis}

\subsection{Observations}

Observations were carried out in two periods, from 13-15 January and 9-19 May 2005 (a total of 14 days), using the Nobeyama Radio Observatory 45-m telescope $\left(H P B W=15^{\prime \prime} 6\right)$ and the 25-channel SIS BEam Array Receiver System (BEARS, Sunada et al. 2000) as the front-end. The observed spectra are ${ }^{13} \mathrm{CO}(J=1-0)(110.201354 \mathrm{GHz})$ and $\mathrm{C}^{18} \mathrm{O}(J=1-0)(109.782178 \mathrm{GHz})$ with observing grid 
spacings of $41^{\prime \prime} 1$ and 10 .'3, respectively. The receiver back-end has a $32 \mathrm{MHz}$-bandwidth, 1024-channel digital spectrometer, resulting in a velocity resolution of about $0.1 \mathrm{~km} \mathrm{~s}^{-1}$. All observations were carried out in position-switching mode. The standard chopper-wheel method was used to convert the received intensity into the antenna temperature, $T_{\mathrm{A}}^{*}$. To convert to radiative temperature from antenna temperature, we used 0.45 at $112 \mathrm{GHz}$ as the value of the main beam efficiency. The total observation time was $80 \mathrm{~h}$. We observed the entire W5-East region $\left(1.0^{\circ} \times 0.6^{\circ}\right)$ in ${ }^{13} \mathrm{CO}(J=1-0)$ in the first period, and detected clouds (total area: $\left.0.16 \mathrm{deg}^{2}\right)$ in $\mathrm{C}^{18} \mathrm{O}(J=1-0)$ in the second period. The coordinates of the FOV center for ${ }^{13} \mathrm{CO}$ are $(l, b)=(138.0,1.5)$. We used off position: $(l, b)=(138.5,0.9)$ during the entire observation period. To correct for beam-to-beam variation, we calibrated the intensity scale of each beam using a $100 \mathrm{GHz}$ SIS receiver (S100) with an SSB filter. Pointing was checked every $100 \mathrm{~min}$ by observing an $\mathrm{SiO}$ maser point source. The estimated pointing accuracy was about $5^{\prime \prime}$ on average. The system noise temperature $\left(T_{\text {sys }}\right)$ was $450 \mathrm{~K}$ for both lines on average.

\subsection{Data reduction}

For data reduction, we used "Newstar" provided by the Nobeyama Radio Observatory. In the Newstar process, the positional distortions of the 25 beams of BEARS were corrected using scaling and regridding tasks. In addition, the baseline was subtracted from each spectrum by linear function fitting, taking $6 \mathrm{~km} \mathrm{~s}^{-1}$ wide emission-free portions as the continuum.

\subsection{Physical parameter estimations}

We calculated the column densities from the observational flux at each observed point. Each molecular cloud observed in W5-East is much larger than the beam-size of the NRO $45-\mathrm{m}$ telescope (15'.6); thus, we assumed that the beam filling factor was unity. To calculate the column density, we assumed a uniform excitation temperature $\left(T_{\mathrm{ex}}\right)$ of $30 \mathrm{~K}$ in all molecular clouds. This assumption is based on the dust temperature derived from 2-mm continuum observations of BRCs carried out by Sugitani et al. (2000). For the typical case of 7.6-16 K peak antenna temperature, the variations in $T_{\mathrm{ex}} \pm 10 \mathrm{~K}$ give a $10 \%$ error in column density. By assuming local thermodynamic equilibrium (LTE), the optical depth $\left(\tau_{v}\right)$ and molecular column density $(N)$ are given as follows:

$\tau_{v}=-\ln \left(1-\frac{k T_{\mathrm{R}}^{*}}{h v}\left(\frac{1}{\exp \left(h v / k T_{\mathrm{ex}}\right)}-\frac{1}{\exp \left(h v / k T_{\mathrm{BB}}\right)}\right)^{-1}\right)$

$N=\frac{3 k T_{\mathrm{ex}}}{8 \pi^{3} B \mu_{\mathrm{d}}^{2}} \frac{\int \tau_{\nu} \mathrm{d} v}{1-\exp \left(-h v / k T_{\mathrm{ex}}\right)}$,

where $T_{\mathrm{R}}^{*}$ is the radiative temperature obtained from observation, $h$ is the Plank constant, $k$ is the Boltzmann constant, $v$ is the observed rest frequency, $T_{\mathrm{ex}}$ is the excitation temperature of the molecular clouds, $T_{\mathrm{BB}}$ is the temperature of the cosmic background radiation $(2.7 \mathrm{~K}), B$ is the rotational constant, and $\mu_{\mathrm{d}}$ is the dipole moment of the $\mathrm{CO}$ molecule. In the case of ${ }^{13} \mathrm{CO}$, $v=110.201354 \mathrm{GHz}, \mu_{\mathrm{d}}=0.112$ Debye, $B=55.10 \mathrm{GHz}$. In the case of $\mathrm{C}^{18} \mathrm{O}, v=119.782182 \mathrm{GHz}, \mu_{\mathrm{d}}=0.112$ Debye and $B=55.10 \mathrm{GHz}$.

To convert from ${ }^{13} \mathrm{CO}$ and $\mathrm{C}^{18} \mathrm{O}$ column densities to $\mathrm{H}_{2}$ column density, we need to know the molecular abundance relative to $\mathrm{H}_{2}$. This, however, differs from the terrestrial abundance, depending on circumstances. Thus, we formulated new column density conversion equations from $N\left({ }^{13} \mathrm{CO}\right)$ and $N\left(\mathrm{C}^{18} \mathrm{O}\right)$ to $N\left(\mathrm{H}_{2}\right)$ in this region. The conversion function is as follows:

$N\left(\mathrm{H}_{2}\right)=\left[\frac{N\left({ }^{13} \mathrm{CO}\right)}{2.7 \times 10^{15}}+0.6\right] \times 10^{21}$
$N\left(\mathrm{H}_{2}\right)=\left[\frac{N\left(\mathrm{C}^{18} \mathrm{O}\right)}{1.7 \times 10^{14}}-0.8\right] \times 10^{21}$

(see Appendix). The projected area $(A)$ is obtained by counting the spatial elements of observed points where the integrated intensity satisfied our criteria as a molecular cloud (For example, in the case of ${ }^{13} \mathrm{CO}$, molecular clouds with boundaries of $5 \sigma$ integrated intensity). The area element is defined as a square of observed grid spacings. The grid spacings are 41". 1 and 10.'3 for $13 \mathrm{CO}$ and $\mathrm{C} 18 \mathrm{O}$, respectively. The mass of each cloud and clump $\left(M_{\mathrm{LTE}}\right)$ is defined as:

$M_{\mathrm{LTE}}=\left(\Sigma N\left(\mathrm{H}_{2}\right) \times m_{\mathrm{H}} \times \mu \times A\right)$,

where the mass of atomic hydrogen $\left(m_{\mathrm{H}}\right)$ and mean molecular weight $(\mu)$ are $m_{\mathrm{H}}=1.660538 \times 10^{-24} \mathrm{~g}$ and $\mu=2.53$, respectively. To calculate number densities, we assumed that each clump is spherical with a uniform density whose effective radius $r$ is defined as:

$r=\sqrt{\frac{A}{\pi}}$

\section{Results}

\section{1. ${ }^{13} \mathrm{CO}(\mathrm{J}=1-0)$ and molecular clouds}

Figure 1 is the integrated intensity map of ${ }^{13} \mathrm{CO}(J=1-0)$. The orange contours illustrate ${ }^{13} \mathrm{CO}(J=1-0)$ line integrated intensity superposed on the Digitized Sky Survey (DSS) R-band image around W5-East in galactic coordinates. The integrated velocity ranges run from $-44 \mathrm{~km} \mathrm{~s}^{-1}$ to $-34 \mathrm{~km} \mathrm{~s}^{-1}$. The ${ }^{13} \mathrm{CO}$ emissions are distributed around the $\mathrm{H}$ II region formed mainly by $\mathrm{BD}+590578$.

We identified eight ${ }^{13} \mathrm{CO}$ molecular clouds with boundaries of $5 \sigma$ integrated intensity, corresponding to $N\left(\mathrm{H}_{2}\right)=6.0 \times$ $10^{21} \mathrm{~cm}^{-2}\left(A_{\mathrm{V}}=3.2\right.$, Bohlin et al. 1978). They are enumerated as shown in Fig. 1. The masses of the clouds are 460-36000 $M_{\odot}$, with a total of $81000 M_{\odot}$. The physical properties of each cloud, derived according to the procedure described in the previous sections are summarized in Table 1.

In general, the distributions of molecular clouds and dark clouds in the DSS image coincide well. They are concentrated only on the north side of W5-East, forming a semi-arc. In contrast, on the south side of this region, no molecular clouds have been identified. The ${ }^{12} \mathrm{CO}$-emitting clouds (CGPS, Heyer et al. 1998; Wilking et al. 1984) are more extended than the ${ }^{13} \mathrm{CO}$ clouds. For example, the W5NE cloud $(l=137.9, b=1.7)$ observed in ${ }^{12} \mathrm{CO}$ with the MWO 4.9-m telescope (Wilking et al. 1984) consists of five discrete ${ }^{13} \mathrm{CO}$ clouds (clouds $1-5$ ), but little ${ }^{13} \mathrm{CO}$ emission there.

Three molecular clouds associated with BRCs (BRC 12-14) have visible arc-like structures on their edges. The cloud 6 has a simple cometary structure pointing to the exciting star. Its shape appears similar to the morphology predicted by the RDI model (Lefloch \& Lazareff 1994) at $0.21 \times 10^{6}$ years after the beginning of UV radiation. 


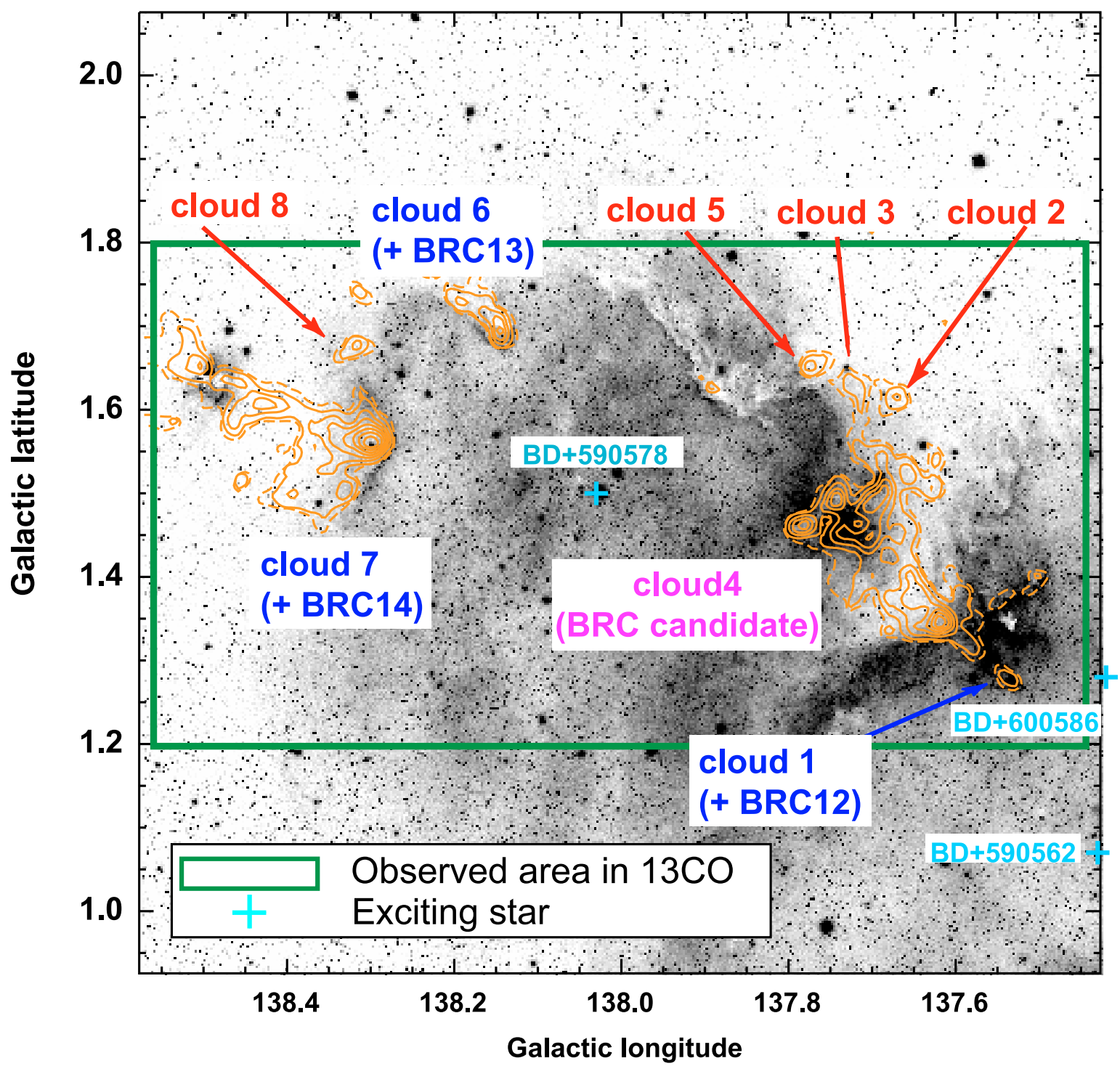

Fig. 1. ${ }^{13} \mathrm{CO}(J=1-0)$ line integrated intensity (orange contour) superposed on the DSS optical image around the W5-East H II region in the galactic coordinates. The dashed contour is $1.725 \mathrm{~K} \mathrm{~km} \mathrm{~s}^{-1}$, corresponding to $3 \sigma$. The solid contours run from $2.725 \mathrm{~K} \mathrm{~km} \mathrm{~s}^{-1}$ (5 $\sigma$; definition border of molecular clouds) with steps of $1.725 \mathrm{~K} \mathrm{~km} \mathrm{~s}^{-1}(3 \sigma)$. The integrated velocity range is from $-44 \mathrm{~km} \mathrm{~s}^{-1}$ to $-34 \mathrm{~km} \mathrm{~s}^{-1}$. We counted eight molecular clouds, associated with (blue) and without (red) BRCs, and cloud 4 (pink), as shown.

The cloud 7 has a head-tail structure like a comet, whereas another small HII region, Sh 2-201, is located in the tail. Sh2-201 did not seem to form as a result of triggering by $\mathrm{BD}+590578$ because it is separated from the visible shock front as the bright rim. Therefore it is suggested that a pre-exciting filamentary cloud spontaneously formed Sh2-201 in the middle, and the west edge of cloud 7 has a dense and massive head delineated by the bright rim. These facts suggest that these molecular clouds are compressed, most likely by UV radiation or stellar winds from BD+590578. Cloud 1 is associated with BRC 12, whose shape is almost spherical. However, BRC 12 is pointing in the direction of the closest early-type star, BD+600586 (O8III). This suggests that cloud 1 is not excited by BD+590578 from outside the main W5-East shell. Cloud 4 is the largest molecular cloud in this region; its intensity peak juts into the W5East region. Arc-like structures similar to BRCs are visible in this part of the cloud. Hereafter, we treat cloud 4 as a molecular cloud surrounded by a bright rim (see also Sect. 4.3). This cloud was not identified as a BRC in previous studies (e.g., Sugitani et al. 1991, see discussions). Four clouds (clouds 2, 3, 5, and 8) without bright rims have almost spherical structures. Note however that, in the Spitzer GLIMPSE $8 \mu \mathrm{m}$, which is more sensitive to ionization fronts, the surface of cloud 8 has a bright rim-like structure. This is not visible in the DSS2 red image. In this paper, cloud 8 is treated as a cloud without a bright rim. It seems to be less affected by UV radiation than the other BRCs. These do not have visible arc-like structures between the molecular cloud and the HII region. This suggests that UV radiation from BD+590578 has little influence on these clouds.

In the previously published ${ }^{12} \mathrm{CO}$ maps, we can find a ridgelike structure in the north of W5-East (e.g., Karr \& Martin 2003). However, we find no ${ }^{13} \mathrm{CO}$ emission in this structure despite the fact that other dark clouds in the visible are well traced by ${ }^{13} \mathrm{CO}$. The sensitivity $(1 \sigma)$ of our ${ }^{13} \mathrm{CO}$ observation is $0.525 \mathrm{~K} \mathrm{~km} \mathrm{~s}^{-1}$ which corresponds to about $N\left(\mathrm{H}_{2}\right)=1.2 \times 10^{21} \mathrm{~cm}^{-2}$. The reason for non-detection in the visible northern ridge structure may be that the shorter distance to BD+590578 (compared to the other dark clouds) result in stronger UV irradiation on the cloud. Thus, the disappearance of ${ }^{13} \mathrm{CO}$ may indicate that ${ }^{13} \mathrm{CO}$ is dissociated from $\mathrm{BD}+590578$ by UV radiation. 
Table 1. Physical parameters at peak $T_{\mathrm{a}}^{*}$ position for each ${ }^{13} \mathrm{CO}$ molecular cloud.

\begin{tabular}{|c|c|c|c|c|c|c|c|c|c|c|c|}
\hline Cloud & $\begin{array}{c}l \\
\text { (degree) }\end{array}$ & $\begin{array}{c}b \\
\text { (degree) }\end{array}$ & $\begin{array}{c}\alpha \\
(\mathrm{J} 2000.0)\end{array}$ & $\begin{array}{c}\delta \\
(\mathrm{J} 2000.0)\end{array}$ & $\begin{array}{c}T_{\mathrm{a}}^{*} \\
(\mathrm{~K})\end{array}$ & $\begin{array}{c}\delta v^{1} \\
\left(\mathrm{~km} \mathrm{~s}^{-1}\right)\end{array}$ & $\begin{array}{c}V_{\mathrm{LSR}^{2}} \\
\left(\mathrm{~km} \mathrm{~s}^{-1}\right)\end{array}$ & $\begin{array}{c}r \\
(\mathrm{pc})\end{array}$ & $\begin{array}{c}N\left(\mathrm{H}_{2}\right) \\
\left(\times 10^{22} \mathrm{~cm}^{-2}\right)\end{array}$ & $\begin{array}{l}\text { Mass } \\
\left(M_{\odot}\right)\end{array}$ & BRC number \\
\hline cloud 1 & 137.53 & 1.28 & 025458 & +603606 & 9.4 & 1.55 & -38.3 & 0.31 & 7.7 & 460 & 12 \\
\hline cloud 2 & 137.67 & 1.62 & 025716 & +605020 & 6.09 & 1.93 & -38.2 & 0.81 & 6.9 & 2700 & - \\
\hline cloud 3 & 137.72 & 1.63 & 025740 & +604928 & 4.66 & 1.41 & -38.8 & 0.71 & 6.8 & 2100 & - \\
\hline cloud 4 & 137.77 & 1.46 & 025723 & +603902 & 16.3 & 1.46 & -39.3 & 2.9 & 8.9 & 36000 & - \\
\hline cloud 5 & 137.77 & 1.65 & 025806 & +604908 & 7.58 & 1.17 & -40.4 & 0.44 & 7.3 & 850 & - \\
\hline cloud 6 & 138.15 & 1.69 & 030100 & +604029 & 11.6 & 1.66 & -38.5 & 1.03 & 8.4 & 4500 & 13 \\
\hline cloud 7 & 138.31 & 1.56 & 030139 & +602902 & 15.1 & 2.27 & -38.1 & 2.8 & 9.4 & 33000 & 14 \\
\hline cloud 8 & 138.32 & 1.68 & 030211 & +603505 & 7.65 & 1.38 & -39.3 & 0.55 & 7.3 & 1300 & - \\
\hline
\end{tabular}

${ }^{1}$ FWHM of the Gaussian fit to the observed spectra.

${ }^{2}$ The velocity corresponding to the peak intensity of the Gaussian fit to the observed spectra.

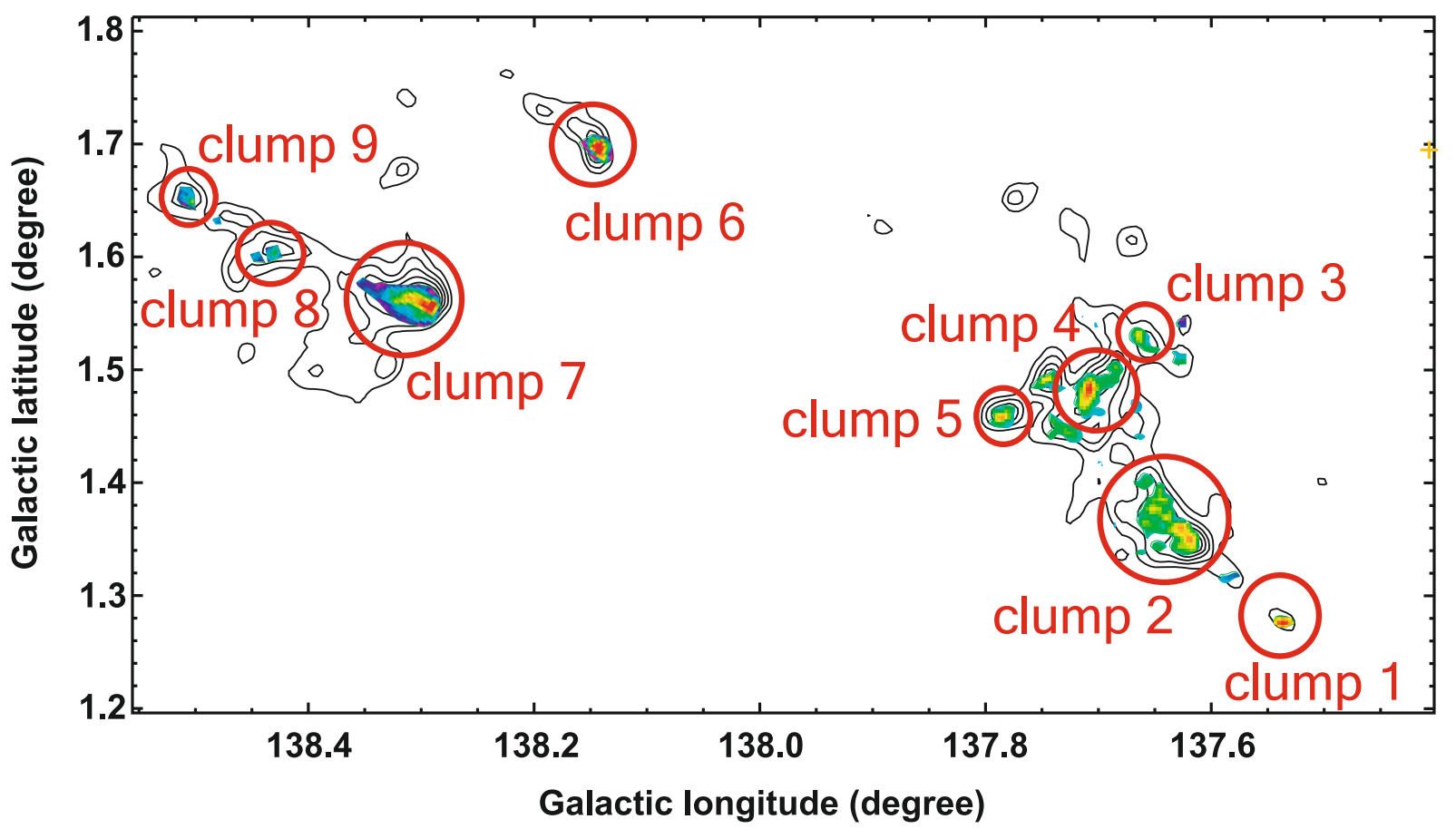

Fig. 2. $\mathrm{C}^{18} \mathrm{O}(J=1-0)$ line integrated intensity map shown by pseudo-color superposed on the ${ }^{13} \mathrm{CO}$ contour map. The lowest contour is $0.66 \mathrm{~K}$ $\mathrm{km} \mathrm{s}^{-1}$ corresponding to $3 \sigma$. The integrated velocity range is the same as ${ }^{13} \mathrm{CO}$.

\section{2. $\mathrm{C}^{18} \mathrm{O}(\mathrm{J}=1-0)$ and clumps}

The pseudo color image in Fig. 2 represents the distribution of the $\mathrm{C}^{18} \mathrm{O}(J=1-0)$ line integrated intensity superposed on the ${ }^{13} \mathrm{CO}(J=1-0)$ map in galactic coordinates. This shows that the $\mathrm{C}^{18} \mathrm{O}$ distribution includes clumpy structures embedded in the ${ }^{13} \mathrm{CO}$ clouds near the intensity peaks. We identified $\mathrm{C}^{18} \mathrm{O}$ clumps using the following procedure:

1. search for the peak position of $\mathrm{C}^{18} \mathrm{O}$ emission in the integrated intensity map;

2 . if the peak integrated intensity is less than $6 \sigma$, stop this procedure;

3. define the extent of the clump by the closed contour of the half-maximum integrated intensity;

4. search for the next-strongest local peak emission outside previously defined clumps;

5. repeat this procedure from (2).

We identified nine clumps. The peak-intensity positions of the clumps show good agreement with ${ }^{13} \mathrm{CO}$ emission peak intensity, even considering the different grid layout between ${ }^{13} \mathrm{CO}$ and $\mathrm{C}^{18} \mathrm{O}$. Following Sect. 2.3, we estimated the physical properties of the clumps. The LTE masses and densities are 55-740 $M_{\odot}$ and $(0.47-2.6) \times 10^{4} \mathrm{~cm}^{-3}$, respectively. The summarized properties of each cloud clump are listed in Table 2.

The distributions of ${ }^{13} \mathrm{CO}$ and $\mathrm{C}^{18} \mathrm{O}$ have a hierarchical density structures; i.e., the $\mathrm{C}^{18} \mathrm{O}$ clumps are embedded in the ${ }^{13} \mathrm{CO}$ clouds, particularly in the region with strong ${ }^{13} \mathrm{CO}$ intensity above $7 \sigma$ (corresponding to $1 \times 10^{22} \mathrm{~cm}^{-2}$ in $N\left(\mathrm{H}_{2}\right)$ ). In clouds $1,4,6$, and $7, \mathrm{C}^{18} \mathrm{O}$ emissions are mostly distributed near the edge of the ${ }^{13} \mathrm{CO}$ molecular cloud. The clouds $2,3,5$, and 8 do not display $\mathrm{C}^{18} \mathrm{O}$ emission. Some of the clumps (clumps 2 , $4,7)$ have cometary head-tail structures. These clumps have large axial ratios and increased density in the direction of the $\mathrm{H}$ II region from inside the ${ }^{13} \mathrm{CO}$ molecular cloud, compared to the other clumps.

We investigated gravitational bounding of $\mathrm{C}^{18} \mathrm{O}$ clumps quantitatively from $M_{\mathrm{LTE}} / M_{\mathrm{vir}}$. The $M_{\mathrm{LTE}} / M_{\mathrm{vir}}$ ratio of clumps 2 , $5,6,7$, and 8 are larger than 1 ; thus, these clumps are gravitationally bound. In particular, we found that all three clumps facing the $\mathrm{H}$ II region (clumps 5, 6, and 7) are gravitationally bounded.

The $\mathrm{C}^{18} \mathrm{O}$ emission in cloud 4 is distributed on each ${ }^{13} \mathrm{CO}$ local peak. On the north side of cloud 4 (around clumps 4 and 5), 


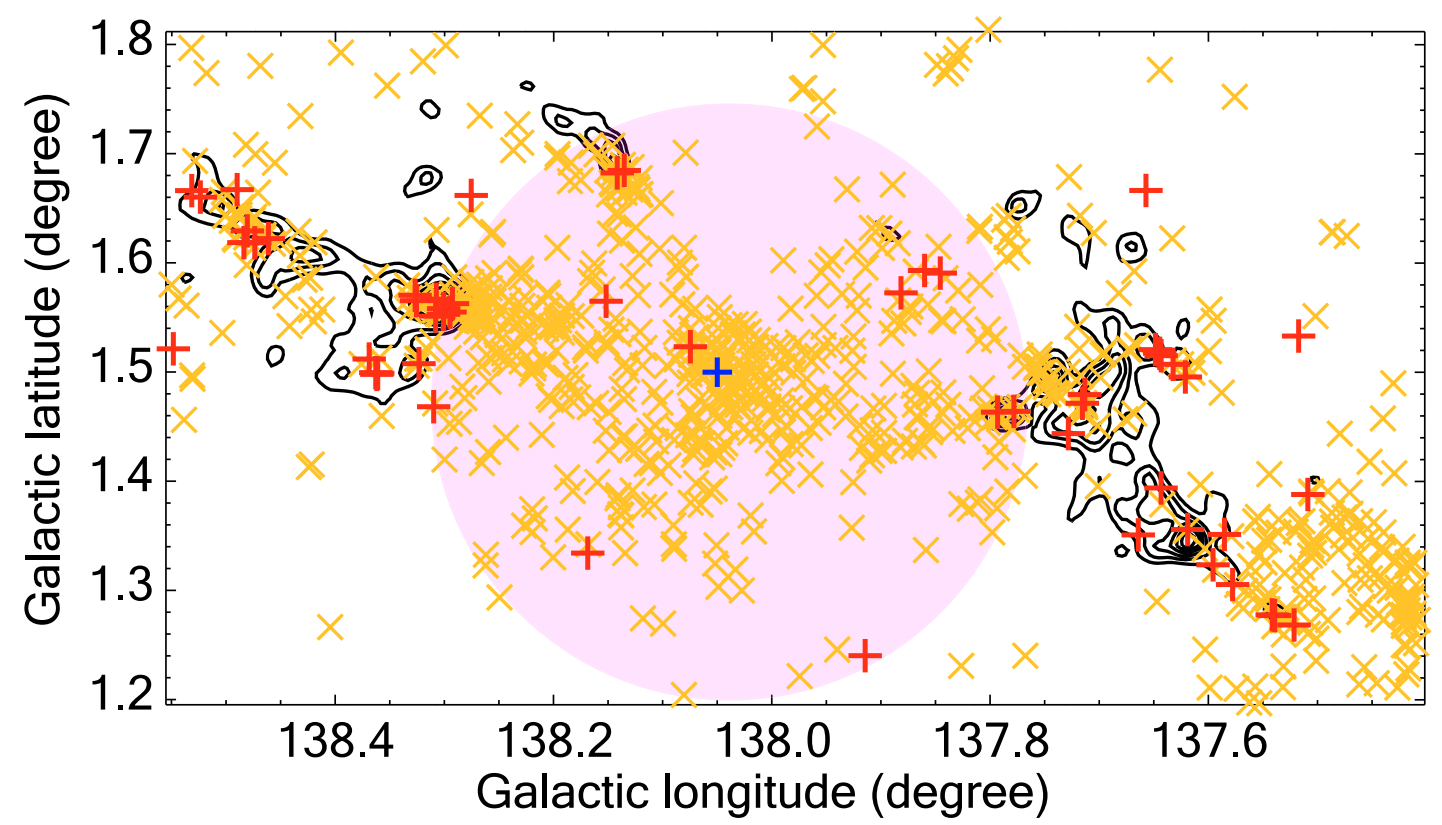

Fig. 3. Spatial distributions of the YSO candidates. The pink circles and blue plus symbols indicate the approximate extent of the H II region seen in the DSS image and the exciting star BD+590578, respectively. The orange crosses and red plus symbols correspond to Class II and Class I candidates, respectively, identified by Koenig et al. (2008) from Spitzer IRAC/MIPS observations, respectively. The black contours represent ${ }^{13} \mathrm{CO}$ molecular clouds from $5 \sigma$ with a $3 \sigma$ step.

Table 2. Physical parameters of $\mathrm{C}^{18} \mathrm{O}(J=1-0)$ clumps.

\begin{tabular}{|c|c|c|c|c|c|c|c|c|c|c|c|c|c|c|}
\hline Clump & $\begin{array}{c}l^{1} \\
\text { (degree) }\end{array}$ & $\begin{array}{c}b^{1} \\
\text { (degree) }\end{array}$ & $\begin{array}{c}\alpha^{1} \\
(\mathrm{~J} 2000.0)\end{array}$ & $\begin{array}{c}\delta^{1} \\
(\mathrm{~J} 2000.0)\end{array}$ & $\begin{array}{c}T_{\mathrm{a}}^{*} \\
(\mathrm{~K})\end{array}$ & $\begin{array}{c}\delta v^{2} \\
\left(\mathrm{~km} \mathrm{~s}^{-1}\right)\end{array}$ & $\begin{array}{c}V_{\mathrm{LSR}} \\
\left(\mathrm{km} \mathrm{s}^{-1}\right)\end{array}$ & $\begin{array}{c}r \\
(\mathrm{pc})\end{array}$ & $\begin{array}{c}N\left(\mathrm{H}_{2}\right) \\
\left(\times 10^{22} \mathrm{~cm}^{-2}\right)\end{array}$ & $\begin{array}{c}n\left(\mathrm{H}_{2}\right) \\
\left(\times 10^{4} \mathrm{~cm}^{-3}\right)\end{array}$ & $\begin{array}{c}\text { LTE mass } \\
\left(M_{\mathrm{LTE}}\right)\left(M_{\odot}\right)\end{array}$ & $\begin{array}{l}\text { Virial mass } \\
\left(M_{\text {vir }}\right)^{4}\left(M_{\odot}\right)\end{array}$ & $M_{\mathrm{LTE}} / M_{\mathrm{vir}}$ & $\begin{array}{c}\text { associated of } \\
{ }^{13} \mathrm{CO} \text { cloud }\end{array}$ \\
\hline 1 & 137.54 & 1.28 & 025503 & +603549 & 0.92 & 1.47 & -38.1 & 0.21 & 2.0 & 1.4 & 55 & 110 & 0.50 & cloud 1 \\
\hline 2 & 137.62 & 1.36 & 025553 & +603721 & 1.11 & 1.54 & -39.3 & 0.85 & 2.3 & 0.47 & 550 & 380 & 1.4 & cloud 4 \\
\hline 3 & 137.66 & 1.53 & 025655 & +604533 & 1.02 & 1.20 & -38.3 & 0.35 & 2.0 & 1.2 & 67 & 85 & 0.79 & cloud 4 \\
\hline 4 & 137.71 & 1.48 & 025701 & +604146 & 1.32 & 1.50 & -38.7 & 0.61 & 3.0 & 1.3 & 170 & 180 & 0.94 & cloud 4 \\
\hline 5 & 137.78 & 1.46 & 025727 & +603846 & 0.91 & 0.96 & -39.3 & 0.33 & 3.7 & 0.97 & 110 & 68 & 1.6 & cloud 4 \\
\hline 6 & 138.14 & 1.69 & 030056 & +604046 & 1.38 & 1.26 & -38.1 & 0.46 & 3.3 & 1.5 & 230 & 130 & 1.8 & cloud 6 \\
\hline 7 & 138.29 & 1.55 & 030128 & +602905 & 2.60 & 1.79 & -38.3 & 0.84 & 5.8 & 1.8 & 740 & 370 & 2.0 & cloud 7 \\
\hline 8 & 138.43 & 1.60 & 030240 & +000000 & 1.50 & 1.33 & -38.6 & 0.39 & 3.3 & 1.5 & 180 & 140 & 1.3 & cloud 7 \\
\hline 9 & 138.50 & 1.65 & 030321 & +602817 & 1.94 & 1.68 & -37.9 & 0.39 & 4.3 & 2.6 & 130 & 160 & 0.81 & cloud 7 \\
\hline
\end{tabular}

${ }^{1}$ The coordinates indicate the peak position. ${ }^{2} F W H M$ of the Gaussian fit to the observed spectra. ${ }^{3}$ The velocity corresponding to the peak intensity of the Gaussian fit to the observed spectra. ${ }^{4} M_{\text {vir }}=210 \times r \times \delta v\left(M_{\odot}\right)$.

we found four $\mathrm{C}^{18} \mathrm{O}$-emitting regions, two of which do not satisfy our criteria of (2). In addition, the $V_{\mathrm{LSR}}$ of these clumps are almost the same compared to the ${ }^{13} \mathrm{CO}$ molecular cloud (cloud 4). Therefore, projection effects along the line of sight can be excluded in our study. Three ZAMS were reported by Wilking et al. (1984) in cloud 4, one of them located between clumps 4 and 5 . In addition, clump 4 shows a head-tail structure. This suggests that the ZAMS may affect cloud fragmentation, as will be discussed in Sect. 4.4. Clump 7 in cloud 7 shows a simple head-tail structure. This suggests that clump 7 is not fragmented, even if protostellar objects exist in clump 7. Based on these facts, the compression and evolution processes of clumps seem to vary from cloud to cloud.

\subsection{Spatial distribution of YSO candidates}

To investigate star-formation activities in W5-East through the spatial distribution of molecular clouds and YSO candidates, we used the YSO candidate catalog provided by Koenig et al. (2008). They observed the entire W5 region with the Spitzer IRAC/MIPS and identified 176 Class I candidates and 1806 Class II candidates from the infrared excess of the detected sources based on the slope of the SED: $\alpha_{\mathrm{IR}}=\left(\mathrm{d} \log \left(\lambda F_{\lambda}\right)\right) /(\mathrm{d} \log \lambda)$. They also used near-infrared to mid-infrared $[3.6 \mu \mathrm{m}]-[4.5 \mu \mathrm{m}]$ against $[4.5 \mu \mathrm{m}]-[5.8 \mu \mathrm{m}]$ color-color diagrams to identify reddened stars by their envelopes and disks (for more identification details, see Koenig et al. 2008, Sect. 3.1). We compared the spatial distributions between the molecular clouds and the YSO candidates, and defined an "association" as a YSO candidate overlapping on a particular region (H II regions, molecular clouds, clumps, etc.) on the projected plane of the sky.

Using this criterion, we identified 778 Class II candidates associated with our observed ${ }^{13} \mathrm{CO}$ region. Of the 778 Class II candidates, 115 are associated with the ${ }^{13} \mathrm{CO}$ clouds. The YSO candidates in the entire W5-East are not distributed uniformly but rather form loose clusters. W5-East contains 55 Class I candidates. The Class I candidate distributions are even more clustered. Almost all (40 of 55) objects are concentrated inside the ${ }^{13} \mathrm{CO}$ clouds, especially around the peak positions of integrated intensity. These concentrations (or clusters) will be discussed in Sect. 4.2. 


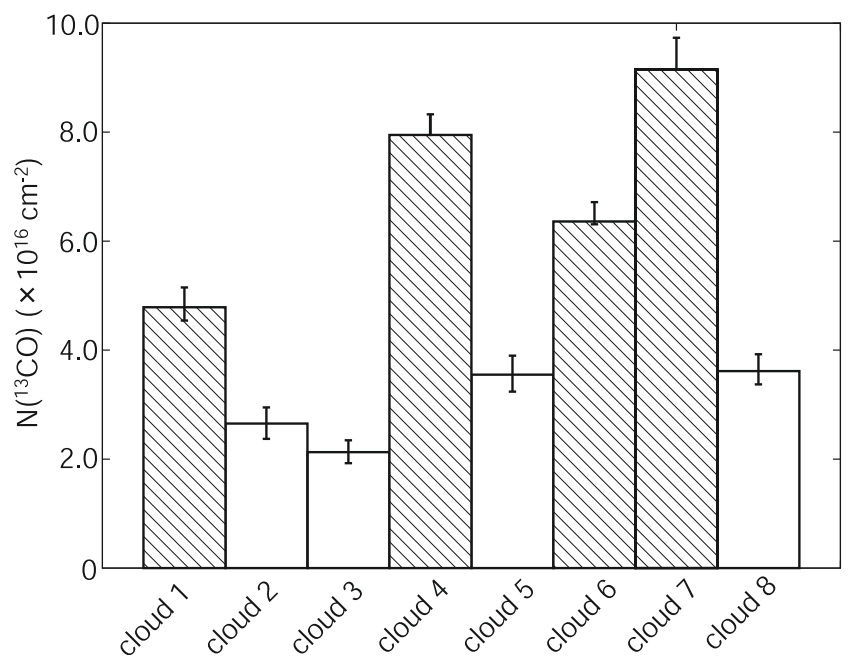

Fig. 4. Comparison of the ${ }^{13} \mathrm{CO}$ peak column densities. The hatched and open bars indicate molecular clouds with and without bright rims, respectively. We assume a uniform excitation temperature of $30 \mathrm{~K}$ with $\pm 5 \mathrm{~K}$ uncertainty shown by error bars. The column densities of the clouds with bright rims are twice as large as the others, suggesting molecular cloud compression.

\section{Discussion}

\subsection{Physical conditions of W5-East}

As shown by our survey, the column density distributions of molecular clouds have steep gradients on the side facing the $\mathrm{H}$ II region. This suggests interaction between the $\mathrm{H}$ II region and the molecular clouds. Figure 4 shows the column densities of molecular cloud peak positions, assuming an excitation temperature of $30 \mathrm{~K}$. The hatched and open bars show those with and without bright rims. The error bars represent the variations in excitation temperature $\pm 5 \mathrm{~K}$. The column densities of ${ }^{13} \mathrm{CO}$ show characteristic differences between the molecular clouds with and without rims. The minimum column density of the cloud with a rim (cloud 1) is 20 percent larger than the maximum column density of the cloud without a rim (cloud 5), and twice as large as the average of clouds $2,3,5$, and 8 . If the excitation temperature variation is less than $10 \mathrm{~K}$, these differences are significant. Together with the facts that the ${ }^{13} \mathrm{CO}$ clouds with rims show steep intensity gradients toward the $\mathrm{H}$ II region, and that the $\mathrm{C}^{18} \mathrm{O}$ clumps are distributed near the head of the cometary cloud structures, the molecular clouds are likely to be compressed by the H II region. Moreover, cloud 4 also has a high column density. This cloud has a visible arc-like structure facing the $\mathrm{H}$ II region, similar to the BRCs.

\subsection{Star-formation activities}

In the previous section, we demonstrated a non-uniform distribution of YSOs. To investigate the distributions of YSO candidates quantitatively, we calculated the surface density of the Class II candidates. We divided the area into square segments of $40^{\prime \prime} \times 40^{\prime \prime}$ (corresponding to the ${ }^{13} \mathrm{CO}$ observation grid spacings) and counted the number of Class II candidates distributed inside (Fig. 5). The surface density contours illustrate that the YSO distribution shows an elongated structure connecting clouds 4 and 7 along the elongation of cloud 4, suggesting a past filamentary GMC that formed those clusters. To investigate the spatial distributions of the Class II YSOs, we defined "cluster" as a surface density greater than $2.0 \operatorname{arcmin}^{-2}\left(2.3 \mathrm{pc}^{-3}\right)$ corresponding to the green contour in Fig. 5. Following our criteria, in W5-East, we see eight loose clusters of Class II candidates near boundaries between the $\mathrm{H}$ II region and the bright rims (BRC 12-14 and cloud 4), around BD+590578, and around the east part of cloud 7 (Sh 2-201). For BRCs, the peak surface densities of the Class II candidates are $11 \mathrm{arcmin}^{-2}$ and above. These two surface-density peaks are shifted toward BD+590578 from each ${ }^{13} \mathrm{CO}$ peak. The same trend can be seen for cloud 4 facing the H II region, although the peak surface density of cloud 4 $\left(7 \operatorname{arcmin}^{-2}\right)$ is lower than that of other BRCs. In addition, AFGL4029 is associated with BRC 14. This object was identified as an ultra-compact H II region by Kurtz et al. (1994) using the Very Large Array (VLA). Their observed spectrum was $2 \mathrm{~cm}$ and $3.6 \mathrm{~cm}$ with a synthesized beam $F W H M$ of $\sim 0.5$ and $\sim 0{ }^{\prime} 9$, respectively. Recently, Zapata et al. (2001) carried out VLA observations of this object at higher spatial resolution (synthesized beam $F W H M \sim 0 ! 2$ ) using the $3.6 \mathrm{~cm}$ continuum. As a result, they found that AFGL4029-IRS1 is a subarcsecond binary separated by $\sim 0$.' 6 . Because BRC 14 has the most massive clump, BRC 14 suggests an active star-forming region.

The surface density of the Class II candidates in cloud 1 is lower than the other BRCs, despite the visible rim structure of BRC 12. Cloud 1 is headed toward the closest early-type star, BD+600586 (O8III), rather than BD+590578. Thus, we do not treat cloud 1 in the same manner as the other BRCs when comparing star-formation activities because cloud 1 is probably excited by BD+600586. The east part of BRC 14 is known as a small H II region, Sh 2-201. This region has a Class II cluster that is independent of the cluster in front of cloud 7. This indicates that Sh 2-201 is another cluster-forming region in the middle of the elongated cloud. In addition, another high surface-density cluster appears in the central region of W5-East (around BD+590578). Its peak surface density is $7 \mathrm{arcmin}^{-2}$, comparable to BRC 14 and cloud 4, but the extent of the cluster is larger. This cluster has only one Class I candidate and no ${ }^{13} \mathrm{CO}$ emission. According to Nakano et al. (2008), the age of these sources is about $4 \mathrm{Myr}$, older than those on the front side of cloud 7 (1 Myr). Therefore, they concluded that these sources are possibly the "first generation cluster", formed together with $\mathrm{BD}+590578$.

The Class I candidates for the cluster-forming clouds are located around the ${ }^{13} \mathrm{CO}$ intensity peaks, indicating ongoing star formation. In contrast, Class II candidates are distributed on the front sides of the BRC arcs close to the exciting star. These distributions indicate that these YSO candidates are aligned by age towards the exciting star from molecular clouds. Koenig et al. (2008) observed the W5 H II region using Spitzer IRAC/MIPS and demonstrated the spatial distribution of Class I, II, and III sources. They also pointed out that the ratio of Class II sources to Class I sources in ${ }^{12} \mathrm{CO}$ molecular clouds is about 7 times higher than the ratio of those HII regions in the entire W5. In this section, we attempt to combine our observational results and their results. Most Class I candidates are associated with ${ }^{13} \mathrm{CO}$ intensity peaks, whereas the majority of Class II candidates form clusters around the visible arc-like structure. These indicate YSO age alignments. Therefore, we conclude that this is additional evidence of triggered star formation and our conclusion is supported by Spitzer IRAC/MIPS observations of W5-East.

We also scrutinized the 2MASS/IRAS archive data to identify the spatial distributions of YSOs in W5-East. We identified 18 protostellar IRAS sources and 176 2MASS sources with IR excess as YSO candidates using the color-color diagram for mid- and near-infrared wavelength, respectively (for more 


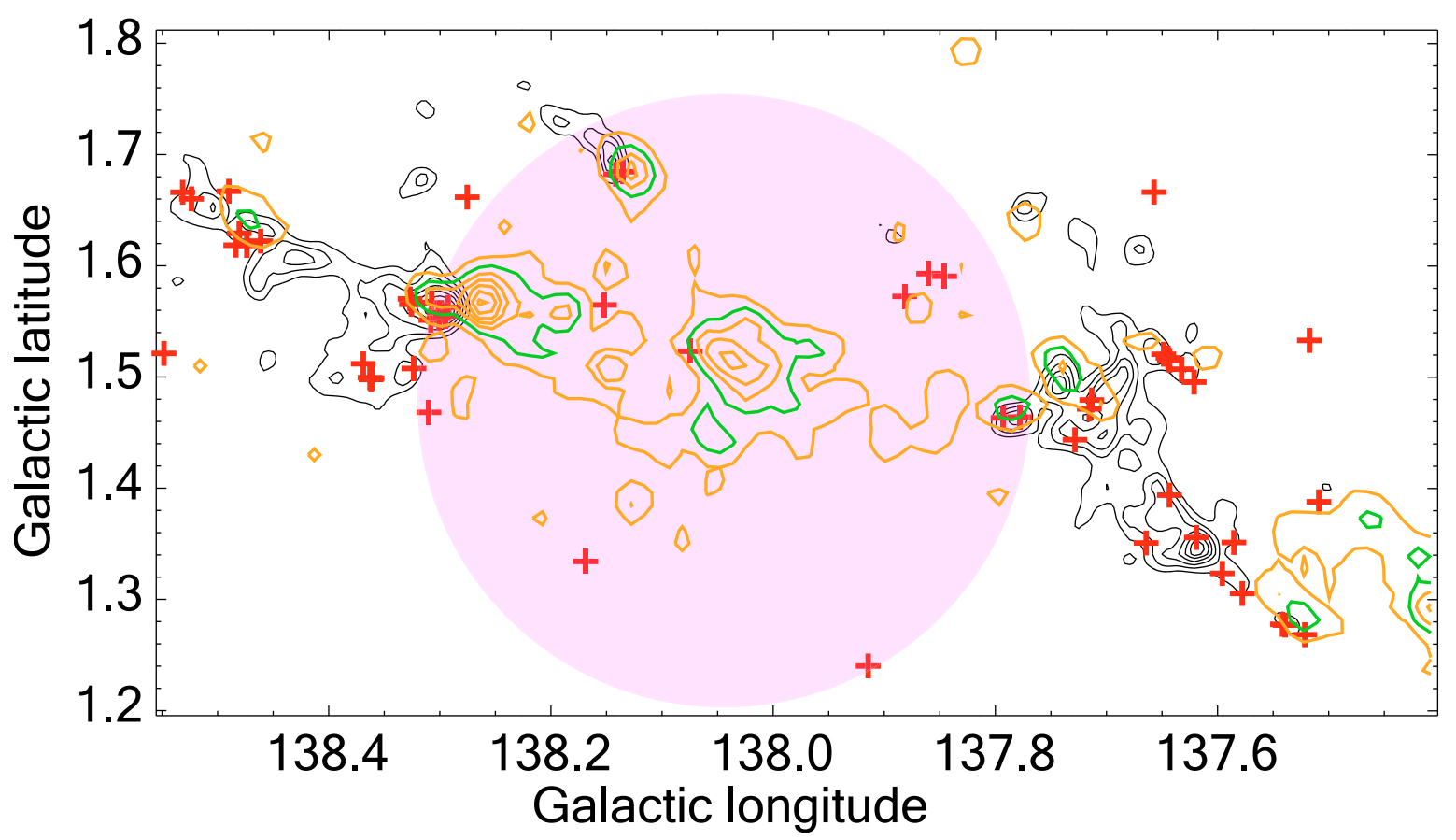

Fig. 5. The orange contours indicate the surface density of the Class II candidates identified by Koenig et al. (2008) with $40 \mathrm{arcsec}^{-2}$ grid resolution, running from $1.0 \mathrm{arcmin}^{-2}$ (corresponding to $1.2 \mathrm{pc}^{-3}$ ) with $1.0 \mathrm{arcmin}^{-2}$ steps. The pink circle, red and blue symbols are same objects as in Fig. 3 . We defined the "cluster" as surface densities greater than $2.0 \operatorname{arcmin}^{-2}\left(2.3 \mathrm{pc}^{-3}\right)$ corresponding to the green contours in this figure. Surface densities of Class II candidates for the front sides of the BRCs, are significantly higher than those of the other regions.

details on the selection rule, see Beichman et al. 1986; Oasa et al. 1999). From these spatial distributions, we found that seven IRAS point sources are located at integrated intensity peaks of ${ }^{13} \mathrm{CO}$, whereas the majority of $2 \mathrm{MASS}$ sources are distributed along the front sides of BRC arcs close to the exciting star. These spatial distributions are very similar to the results of the Spitzer IRAC/MIPS survey, thus triggered star formation in W5-East is supported by both 2MASS/IRAS and IRAC/MIPS observations.

The molecular clouds not facing the H II region (clouds 2, 3, 5, and 8) do not have associated YSO candidates, indicating that no active star formation is taking place. In addition, these clouds do not have visible bright rims and have lower ${ }^{13} \mathrm{CO}$ column densities compared to BRCs. It is possible that UV radiation from W5-East has not yet reached these molecular clouds to trigger cloud compression and star formation.

\subsection{East edge of cloud 4: BRC candidate?}

Even though cloud 4 is not cataloged as a BRC by Sugitani et al. (1991), it exhibits similar properties. The peak ${ }^{13} \mathrm{CO}$ position of cloud 4 is only 1 '.2 from the cloud boundary, close to W5-East. Its column density is three times higher than the average column density of the clouds without bright rims. In addition, Karr \& Martin (2003) pointed out a visible arc-like bright rim on the surface of cloud 4 . The surface density of the Class II candidates has a peak in front of the bright rim associated with cloud 4 , and this peak surface density is similar to that of BRC 14. As mentioned in previous sections, the Class I candidate associated with cloud 4 is unlikely to be a protostellar candidate, but it could suffer from contamination due to high stellar density and poor IRAS resolution. These facts indicate that the east edge of cloud 4 possesses properties similar to clouds 1, 6, and 7. Therefore, we identified this cloud as a "BRC candidate". This BRC candidate shows a complex structure, in contrast to the simple arclike structures of other BRCs (e.g., BRCs 12-14), suggesting different physical conditions in the molecular cloud. However, as one of the possibilities of complex structure, we suggest this may also result from the presence of several different bright rim structures along almost the same line of sight.

\subsection{Column density profiles as indicators of cloud compression}

The cometary morphologies of $\mathrm{BRCs}$ in the ${ }^{13} \mathrm{CO}$ and $\mathrm{C}^{18} \mathrm{O}$ maps indicate that they are compressed from one side, as illustrated in numerical simulations of the RDI model (e.g., Lefloch \& Lazareff 1994). To investigate cloud compression quantitatively, we drew column density profiles of clouds 7 (clump 7), 6 (clump 6) and 4 (clump 5) in Figs. 6-8, respectively. The horizontal axis is the offset distance from the bright rims visible in optical images along the lines connecting the exciting star and the peak positions of ${ }^{13} \mathrm{CO}$ integrated intensity. The solid, dashed and dotted lines are ${ }^{13} \mathrm{CO}, \mathrm{C}^{18} \mathrm{O}$, and the $N\left({ }^{13} \mathrm{CO}\right) / N\left(\mathrm{C}^{18} \mathrm{O}\right)$ ratio, respectively. Note that the $N\left({ }^{13} \mathrm{CO}\right) / N\left(\mathrm{C}^{18} \mathrm{O}\right)$ ratio is calculated only for data points greater than the $3 \sigma$ detection limit in both the ${ }^{13} \mathrm{CO}$ and $\mathrm{C}^{18} \mathrm{O}$ lines.

The column density profile of cloud 7 with BRC 14 in Fig. 6 shows remarkable asymmetry, presenting a sharp cut-off on the side facing the exciting star (front side) and a shallower slope on the opposite side (back side) with peaks at 1 '.5 and $2^{\prime}$ from the bright rim. This trend is almost the same for both ${ }^{13} \mathrm{CO}$ and $\mathrm{C}^{18} \mathrm{O}$. The column density profiles on the back side resemble the power-law function of $N \propto r^{-1}$ for ${ }^{13} \mathrm{CO}$ and are slightly steeper for $\mathrm{C}^{18} \mathrm{O}$. These indicate that molecular gas has undergone compression on the front side due to UV radiation from $\mathrm{BD}+590578$. The $N\left({ }^{13} \mathrm{CO}\right) / N\left(\mathrm{C}^{18} \mathrm{O}\right)$ ratio varies independently of the distance from the shock front. Chemical effects may contribute to the variation of the ${ }^{13} \mathrm{CO} / \mathrm{C}^{18} \mathrm{O}$ relative abundance in a small-scale structure (see Appendix for details). 


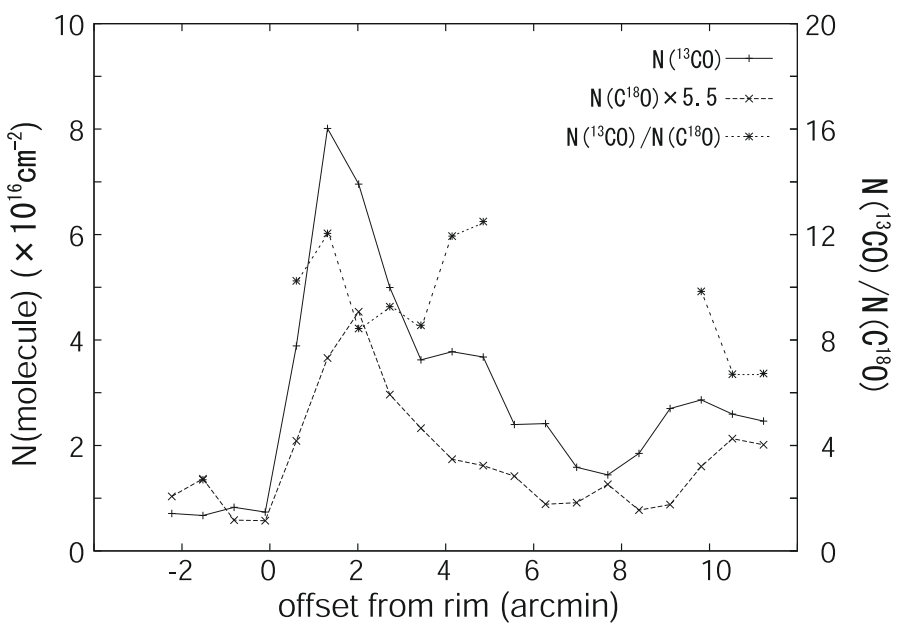

Fig. 6. Column density profile of BRC 14 (cloud 7). The horizontal axis is offset from the bright rim in the optical image. The solid, dashed, and dotted-lines are $N\left({ }^{13} \mathrm{CO}\right), N\left(\mathrm{C}^{18} \mathrm{O}\right) \times 5.5$, and $N\left({ }^{13} \mathrm{CO}\right) / N\left(\mathrm{C}^{18} \mathrm{O}\right)$, respectively.

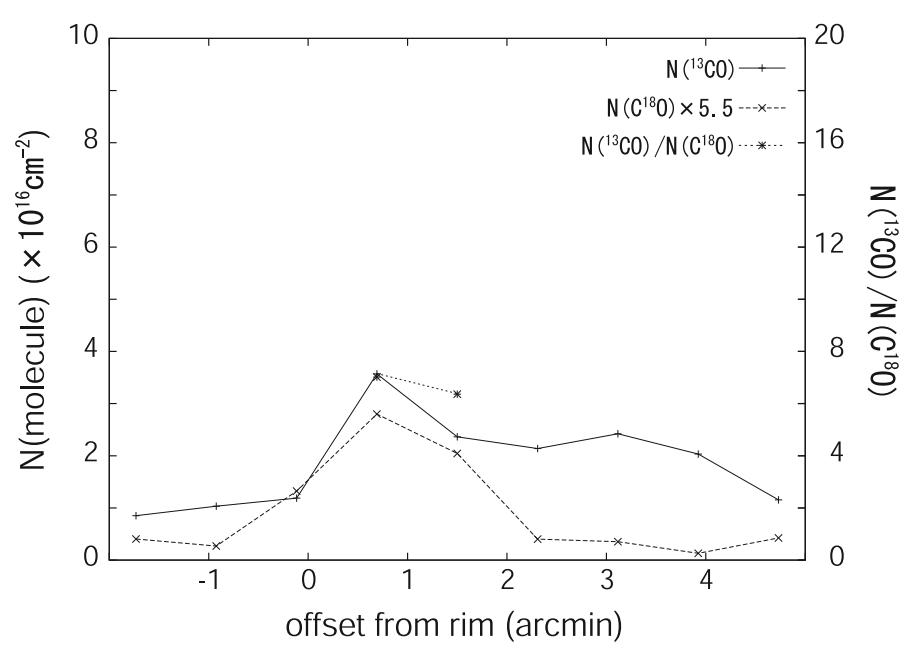

Fig. 7. Column density profile of BRC 13 (cloud 6).

In the case of cloud 6 (Fig. 7), although the trend is less prominent, the same asymmetric feature is seen, particularly for ${ }^{13} \mathrm{CO}$. The profiles have a peak column density at about 0.5 from the bright rim, and the slopes are shallower than those of cloud 7 on both the front and back sides. These indicate compression taking place, although the pressure may be weaker.

For cloud 4, as shown in Fig. 8, clear indications of asymmetry do not appear, even though the $\mathrm{C}^{18} \mathrm{O}$ clump is slightly offset from the rim. The $\mathrm{C}^{18} \mathrm{O}$ intensity is relatively weak, as denoted by the high $N\left({ }^{13} \mathrm{CO}\right) / N\left(\mathrm{C}^{18} \mathrm{O}\right)$ ratio.

Star formation is triggered as a result of cloud compression by UV radiation and stellar winds emanating from OB stars. In the vicinity of W5-East, we found no indication of a past supernova; thus, we considered the effects of UV radiation and stellar winds from BD+590578. To investigate both effects quantitatively, we calculated kinetic energy and momentum input to nearby clouds.

We first consider UV radiation from OB stars to the molecular cloud. Neutral gas on the cloud surface is photo-evaporated when irradiated by UV light. This ionized gas escapes from the cloud with a velocity of $v_{\mathrm{esc}} \simeq 20 \mathrm{~km} \mathrm{~s}^{-1}$ (Oort \& Spitzer 1955)

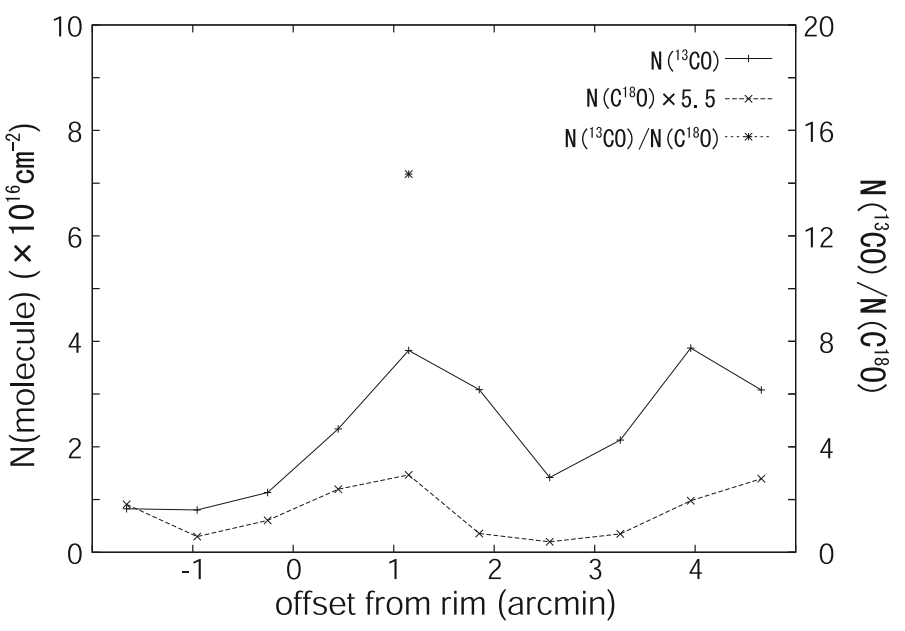

Fig. 8. Column density profile of cloud 4 .

in the rocket effect. The mass loss rate of the molecular cloud $\left(\dot{M}_{\mathrm{UV}}\right)$ is given as

$$
\begin{aligned}
\dot{M}_{\mathrm{UV}}= & 4.0 \times 10^{-5}\left(\frac{N_{\mathrm{Lyman}}}{10^{49} \mathrm{~s}^{-1}}\right)^{1 / 2} \\
& \times\left(\frac{r}{0.1 \mathrm{pc}}\right)^{3 / 2}\left(\frac{d}{1.0 \mathrm{pc}}\right)^{-1} M_{\odot} \mathrm{yr}^{-1}
\end{aligned}
$$

(Stahler \& Palla 2005), where $N_{\text {Lyman }}, r$, and $d$ are the Lyman continuum flux from the exciting star, clump radius, and the distance between the clump and the exciting star, respectively. The kinetic energy $\left(E_{\mathrm{UV}}\right)$ and momentum $\left(P_{\mathrm{UV}}\right)$ are calculated as $E_{\mathrm{UV}}=1 / 2 \times \dot{M}_{\mathrm{UV}} \times v_{\mathrm{esc}}^{2} \times \tau_{\mathrm{age}}$ and $P_{\mathrm{UV}}=\dot{M}_{\mathrm{UV}} \times v_{\mathrm{esc}} \times \tau_{\mathrm{age}}$, respectively, where $\tau_{\text {age }}$ is the time scale of cloud compression.

Stellar wind from a massive star also contributes a significant amount of kinetic energy and momentum to the cloud via highvelocity particles. According to Abbott (1982b), the mass loss rate $\left(\dot{M}_{\mathrm{SW}}\right)$ of a high-mass star caused by stellar wind is given as

$\dot{M}_{\mathrm{SW}}=1.3 \times 10^{16}\left(\frac{L}{L_{\odot}}\right)^{1.77} M_{\odot} \mathrm{yr}^{-1}$,

where $L$ is the luminosity of the star. We assume that the stellar wind is uniformly driven and the energy and momentum input is proportional to the solid angle ratio $(\Omega)$ of the molecular cloud and the spherical $\mathrm{H}$ II region as

$\Omega=\frac{\pi r^{2}}{4 \pi d^{2}}$.

Thus, the cloud obtains kinetic energy $\left(E_{\mathrm{SW}}\right)$ and momentum $\left(P_{\mathrm{SW}}\right)$ from the stellar wind as $E_{\mathrm{SW}}=1 / 2 \dot{M}_{\mathrm{SW}} \times v_{\text {term }}^{2} \times \Omega \times$ $\tau_{\text {age }}$ and $P_{\mathrm{SW}}=\dot{M}_{\mathrm{SW}} \times v_{\text {term }} \times \Omega \times \tau_{\text {age }}$, respectively. The $v_{\text {term }}$ represents the terminal velocity of stellar wind, $\sim 3000 \mathrm{~km} \mathrm{~s}^{-1}$ (Abbott 1982a).

$\mathrm{BD}+590578$ 's spectral type is O7V, and the Lyman photon number and stellar luminosity are given as $\log \left(N_{\text {Lyman }}\right)=$ $48.86 \mathrm{~s}^{-1}$ and $\log \left(L / L_{\odot}\right)=5.24$, respectively (Panagia 1973). We set a cylindrical molecular cloud with a cross section of $\pi r^{2}\left(\sim 0.2 \mathrm{pc}^{2}\right)$ subtending the exciting star located on the surface of the spherical H II region at a distance of $d=15 \mathrm{pc}$ from the star. These quantities correspond to those of clump 6 (BRC 13).

As indicated by the association with the Class-II candidates, $\tau_{\text {age }}$ is estimated to be $\sim 10^{6} \mathrm{yr}, \sim 1 / 4$ of the lifetime of an O7type star. Under these assumptions, we calculated the cumulative amount of energy and momentum contributed to the cloud 
over this timescale. The kinetic energy of the UV radiation $\left(E_{\mathrm{UV}}\right)$ and stellar wind $\left(E_{\mathrm{SW}}\right)$ were calculated as $9 \times 10^{46} \mathrm{erg}$ and $5 \times 10^{45} \mathrm{erg}$, respectively. The momentum of UV radiation $\left(P_{\mathrm{UV}}\right)$ and stellar wind $\left(P_{\mathrm{SW}}\right)$ are $9 \times 10^{40} \mathrm{~g} \mathrm{~cm} \mathrm{~s}^{-1}$ and $3 \times 10^{37} \mathrm{~g} \mathrm{~cm} \mathrm{~s}^{-1}$, respectively. These comparisons indicate that the UV-radiation contributions to kinetic energy $\left(E_{\mathrm{UV}}\right)$ and momentum $\left(P_{\mathrm{UV}}\right)$ are one and three orders of magnitude larger (respectively) than those of the stellar wind $\left(E_{\mathrm{SW}}\right.$ and $\left.P_{\mathrm{SW}}\right)$. Thus, we conclude that UV radiation is the dominant source of molecular cloud compression. Given this condition, $\dot{M}_{\mathrm{UV}}$ is calculated to be $\sim 7 \times 10^{-5} M_{\odot} \mathrm{yr}^{-1}$ cloud 6 can survive more than $6 \times 10^{7} \mathrm{yr}$. For small cloud 5, $\dot{M}_{\mathrm{UV}}=2.5 \times 10^{-5} M_{\odot} \mathrm{yr}^{-1}$; the cloud would photo-evaporate in $3.5 \times 10^{7} \mathrm{yr}$. These molecular cloud "lifetimes" are about an order of magnitude longer than that of the exciting star, BD+590578. This fact indicates that these molecular clouds will be exposed to the destructive shock wave of a supernova explosion at the end of the lifetime of BD+590578, dissipating all the molecular clouds.

We estimated the internal kinetic energy and momentum of the molecular cloud as $E_{\text {kin }}=\frac{1}{2} M_{\text {cloud }} \sigma^{2}$ and $p=M_{\text {cloud }} \sigma$ from the cloud mass $M_{\text {cloud }}$ and velocity dispersion $\sigma(=\Delta V / \sqrt{8 \ln 2})$. For cloud $6, E_{\text {kin }}$ and $P$ are estimated to be $2 \times 10^{46} \mathrm{erg}$ and $6 \times 10^{41} \mathrm{~g} \mathrm{~cm}^{-1}$, respectively. $E_{\text {kin }}$ is comparable to $E_{\mathrm{UV}}$, indicating that external compression can overcome internal pressure and initiate the dynamical collapse of the cloud. Note, however, that in our estimation, we assumed that the energy conversion was $100 \%$ efficient, thus, the energy input may be over-estimated. Additionally, $M_{\text {cloud }}$ is derived from ${ }^{13} \mathrm{CO}$ observations and the mass of the diffuse low-density gas should be taken into account. The internal energy of the molecular cloud is therefore underestimated. Diffuse gas streaming motion was observed in ${ }^{12} \mathrm{CO}$ in the nearby H II region (Tachihara et al. 2000a). From cloud energetics calculations, they concluded that UV radiation is the main energy source, similar to the present study. Our observations suggest that UV radiation plays an important role in molecular cloud compression on the surface as modeled by RDI.

\section{Conclusions}

We carried out a high spatial resolution survey of the entire W5-East $\mathrm{H}$ II region in ${ }^{13} \mathrm{CO}(J=1-0)$ and $\mathrm{C}^{18} \mathrm{O}(J=1-0)$ to investigate physical conditions and star-formation activities in the molecular clouds around the H II region. Our main conclusions are as follows.

1. From the ${ }^{13} \mathrm{CO}(J=1-0)$ survey results, eight molecular clouds were identified, three of which are associated with BRCs. Total mass is $81000 M_{\odot}$. In the $\mathrm{C}^{18} \mathrm{O}(\mathrm{J}=$ $1-0)$ search, nine clumps were identified. These average LTE masses and number densities are 55-740 $M_{\odot}$ and $(0.47-2.6) \times 10^{4} \mathrm{~cm}^{-3}$, respectively.

2. From molecular cloud column density comparisons, we found that molecular clouds with bright rims have column densities twice as high as others in ${ }^{13} \mathrm{CO}(J=1-0)$. This suggests that the molecular clouds are compressed by the H II region.

3. To investigate star-formation activities, we selected the Class I and Class II candidates from previous Spitzer IRAC/MIPS observational results. Forty Class I candidates are located around ${ }^{13} \mathrm{CO}$ cloud integrated intensity peaks, whereas the majority of the Class II candidates form clusters that are distributed on the front sides of the BRC arcs close to the exciting star. This suggests sequential alignment of YSO candidates and molecular clouds in order of age. Thus, our observational results support the idea of triggered star formation in the W5-East $\mathrm{H}$ II region.

4. The intensity peak of the cloud 4 (clump 5) juts into W5-East and exhibits properties similar to the BRCs. Thus, we identified cloud 4 as a BRC candidate for this region.

5. To discuss the dominant triggering factor, we quantitatively considered the effects of UV radiation and stellar winds from $\mathrm{BD}+590578$. UV radiation contributions to kinetic energy and momentum are one and three orders of magnitude larger than those from the stellar wind. Therefore, we concluded that the dominant factor in triggering in the W5-East region was UV radiation.

6. The cometary shapes of BRCs were investigated in terms of column density profiles. They exhibit sharp edges on the side facing the $\mathrm{H}$ II region, and the backs have a density gradient resembling material compressed by UV radiation (e.g., Lefloch \& Lazareff 1994).

Acknowledgements. These observations were carried out as part of the "Education Program for Graduate Students" organized by the Nobeyama Radio Observatory (NRO). The NRO is a branch of the National Astronomical Observatory of Japan, National Institutes of Natural Sciences. We thank the NRO staff for supporting our observations. This work was supported by the "21st Century COE Program: The Origin and Evolution of Planetary Systems" of the Ministry of Education, Culture, Sports, Science, and Technology (MEXT), Japan. We are grateful to Xavier Koenig and Lori Allen for providing the source catalog for the Spitzer Space Telescope. The provided Spitzer Space Telescope data set greatly improved the precision and clarity of our discussion. We also thank Yuri Aikawa and Makoto Nakano for fruitful discussions.

\section{Appendix A: Conversion functions from $N\left({ }^{13} \mathrm{CO}\right)$ and $N\left(\mathrm{C}^{18} \mathrm{O}\right)$ into $N\left(\mathrm{H}_{2}\right)$}

To estimate the column densities of $\mathrm{H}_{2}$, we need to know the abundance ratio of ${ }^{13} \mathrm{CO}$ and $\mathrm{C}^{18} \mathrm{O}$ versus $\mathrm{H}_{2}$.

First, to investigate the relationships between ${ }^{13} \mathrm{CO}$ and $\mathrm{C}^{18} \mathrm{O}$, we selected observed points with a ${ }^{13} \mathrm{CO}$ and $\mathrm{C}^{18} \mathrm{O}$ separation of less than $5^{\prime \prime}$. Figure A.1 is the correlation diagram of the column densities of ${ }^{13} \mathrm{CO}$ and $\mathrm{C}^{18} \mathrm{O}$, showing a poor correlation between them. This implies that the relative abundance of ${ }^{13} \mathrm{CO}$ and $\mathrm{C}^{18} \mathrm{O}$ changes with location. The maximum abundance ratio $\left[N\left({ }^{13} \mathrm{CO}\right)\right] /\left[N\left(\mathrm{C}^{18} \mathrm{O}\right)\right]$ is about 20 , much larger than the terrestrial abundance ratio (5.5). This cannot be explained on the basis of simple optical depth arguments, but must include chemical effects, such as selective destruction by UV radiation, molecular depletion, and ion-exchange reactions (e.g. Frerking et al. 1982; Glassgold et al. 1985). We cannot, however, see any characteristic spatial distribution in abundance variation, i.e., there is no abundance anomaly distributed only on the molecular cloud close to the $\mathrm{H}$ II region. The column density profile of ${ }^{13} \mathrm{CO}$ and $\mathrm{C}^{18} \mathrm{O}$ (see Sect. 4.5) indicates this. In addition, we cannot see component abundance variation in our line of sight. Therefore, to estimate the column density of $\mathrm{H}_{2}$, we did not consider the abundance variation with location. We used uniform relationships between ${ }^{13} \mathrm{CO}$ and $\mathrm{C}^{18} \mathrm{O}$. To obtain the relationships, we derived a correlation between the $N\left({ }^{13} \mathrm{CO}\right)$ and $N\left(\mathrm{C}^{18} \mathrm{O}\right)$ by least-square fitting. The column density relationships are formulated by the following equation:

$N\left({ }^{13} \mathrm{CO}\right)=15.7 \times N\left(\mathrm{C}^{18} \mathrm{O}\right)-3.7 \times 10^{15}$.

Comparing this relation with that of other regions (Fig. A.1), our result approximates that of Ophiuchus North derived by Tachihara et al. (2000b). Thus we adopt the ${ }^{13} \mathrm{CO}$ to $\mathrm{H}_{2}$ 


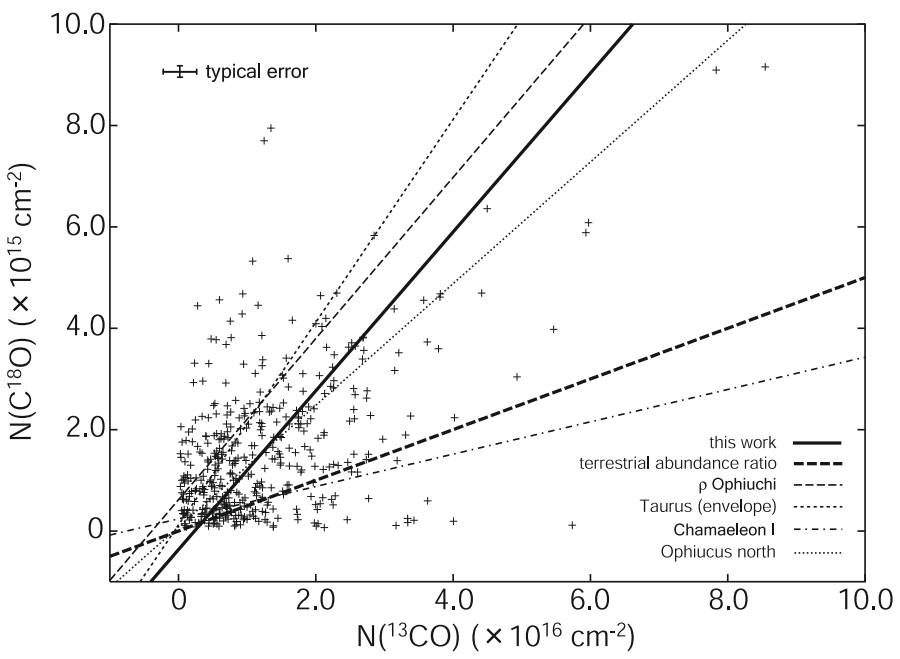

Fig. A.1. Comparison of detected ${ }^{13} \mathrm{CO}$ and $\mathrm{C}^{18} \mathrm{O}$ column densities (crosses). The solid line and other lines represent the best-fit function by least-square fitting in this work and previous studies, respectively. The results of $\rho$ Ophiuchi and Taurus (envelope) are calculated by Frerking et al. (1982). The Chamaeleon I and Ophiuchus North cloud complexes were studied by Hayakawa et al. (1999), Nozawa et al. (1991) and Tachihara et al. (2000b), respectively.

conversion function derived in Ophiuchus North by Nozawa et al. (1991) as follows:

$N\left(\mathrm{H}_{2}\right)=\left[\frac{N\left({ }^{13} \mathrm{CO}\right)}{2.7 \times 10^{15}}+0.6\right] \times 10^{21}$.

Therefore, for $\mathrm{C}^{18} \mathrm{O}$, we adopted Eq. (A.1) to Eq. (A.2) and derived a new conversion function in W5-East:

$N\left(\mathrm{H}_{2}\right)=\left[\frac{N\left(\mathrm{C}^{18} \mathrm{O}\right)}{1.7 \times 10^{14}}-0.8\right] \times 10^{21}$.

We used these conversion functions to estimate the physical parameters of the molecular clouds and cloud clumps in this paper.

\section{References}

Abbott, D. C. 1982a, ApJ, 263, 723

Abbott, D. C. 1982 b, ApJ, 259, 282

Beichman, C. A., Myers, P. C., Emerson, J. P., et al. 1986, ApJ, 307, 337
Bohlin, R. C., Savage, B. D., \& Drake, J. F. 1978, ApJ, 224, 132 Carpenter, J. M., Heyer, M. H., \& Snell, R. L. 2000, ApJS, 130, 381 Deharveng, L., Lefloch, B., Massi, F., et al. 2006, A\&A, 458, 191

Deharveng, L., \& Zavagno, A. 2008, in Massive Star Formation: Observations Confront Theory, ed. H. Beuther, H. Linz, \& T. Henning, ASP Conf. Ser., 387,338

Deharveng, L., Zavagno, A., \& Caplan, J. 2005, A\&A, 433, 565

Elmegreen, B. G., \& Lada, C. J. 1977, ApJ, 214, 725

Frerking, M. A., Langer, W. D., \& Wilson, R. W. 1982, ApJ, 262, 590

Georgelin, Y. M., \& Georgelin, Y. P. 1976, A\&A, 49, 57

Glassgold, A. E., Huggins, P. J., \& Langer, W. D. 1985, ApJ, 290, 615

Hayakawa, T., Mizuno, A., Onishi, T., et al. 1999, PASJ, 51, 919

Heyer, M. H., Brunt, C., Snell, R. L., et al. 1998, ApJS, 115, 241

Karr, J. L., \& Martin, P. G. 2003, ApJ, 595, 900

Koenig, X. P., Allen, L. E., Gutermuth, R. A., et al. 2008, ApJ, 688, 1142

Kurtz, S., Churchwell, E., \& Wood, D. O. S. 1994, ApJS, 91, 659

Lada, C. J., \& Lada, E. A. 2003, ARA\&A, 41, 57

Lefloch, B., \& Lazareff, B. 1994, A\&A, 289, 559

Matsuyanagi, I., Itoh, Y., Sugitani, K., et al. 2006, PASJ, 58, L29

Morgan, L. K., Thompson, M. A., Urquhart, J. S., \& White, G. J. 2008, A\&A, 477,557

Nakano, M., Sugitani, K., Niwa, T., Itoh, Y., \& Watanabe, M. 2008, PASJ, 60

Nozawa, S., Mizuno, A., Teshima, Y., Ogawa, H., \& Fukui, Y. 1991, ApJS, 77, 647

Oasa, Y., Tamura, M., \& Sugitani, K. 1999, ApJ, 526, 336

Oasa, Y., Tamura, M., Nakajima, Y., et al. 2006, AJ, 131, 1608

Ogura, K. 2006, Bull. Astron. Soc. India, 34, 111

Okumura, S.-I., Mori, A., Nishihara, E., Watanabe, E., \& Yamashita, T. 2000, ApJ, 543, 799

Oort, J. H., \& Spitzer, L. J. 1955, ApJ, 121, 6

Panagia, N. 1973, AJ, 78, 929

Shu, F. H. 1977, ApJ, 214, 488

Stahler, S. W., \& Palla, F. 2005, The Formation of Stars, ed. S. W. Stahler, \& F. Palla, ISBN 3-527-40559-3 (Wiley-VCH), 865

Sugitani, K., Fukui, Y., \& Ogura, K. 1991, ApJS, 77, 59

Sugitani, K., Matsuo, H., Nakano, M., Tamura, M., \& Ogura, K. 2000, AJ, 119, 323

Sugitani, K., \& Ogura, K. 1994, ApJS, 92, 163

Sugitani, K., Tamura, M., \& Ogura, K. 1995, ApJ, 455, L39

Sunada, K., Yamaguchi, C., Nakai, N., et al. 2000, in Radio Telescopes, ed. H. R. Butcher, Proc. SPIE, 4015, 237

Tachihara, K., Abe, R., Onishi, T., Mizuno, A., \& Fukui, Y. 2000a, PASJ, 52, 1147

Tachihara, K., Mizuno, A., \& Fukui, Y. 2000b, ApJ, 528, 817

Urquhart, J. S., Thompson, M. A., Morgan, L. K., et al. 2007, A\&A, 467, 1125

Warren, Jr., W. H., \& Hesser, J. E. 1978, ApJS, 36, 497

Whitworth, A. P., \& Zinnecker, H. 2004, A\&A, 427, 299

Wilking, B. A., Doering, C. R., Harvey, P. M., Lada, C. J., \& Joy, M. 1984, ApJ, 279, 291

Yamaguchi, R., Saito, H., Mizuno, N., et al. 1999, PASJ, 51, 791

Zapata, L. A., Rodríguez, L. F., \& Kurtz, S. E. 2001, Rev. Mex. Astron. Astrofis., 37,83 